\title{
Dissipative soliton resonance Ytterbium-doped fiber laser with cylindrical vector beam generation
}

\author{
Jiaojiao Zhang ${ }^{1}$, Zuxing Zhang ${ }^{1, \dagger}$, Yang Xu ${ }^{1}$, Hongdan Wan ${ }^{1}$, Jie Wang ${ }^{1}$, Lin \\ Zhang $^{2}$ \\ ${ }^{1}$ Advanced Photonic Technology Lab, College of Electronic and Optical Engineering, Nanjing \\ University of Posts and Telecommunications, Nanjing 210003, China \\ ${ }^{2}$ Aston Institute of Photonic Technologies, Aston University, Birmingham B4 7ET, UK \\ 'zxzhang@njupt.edu.cn
}

\begin{abstract}
We experimentally demonstrate a dissipative soliton resonance (DSR) Ytterbium-doped fiber laser with high-purity cylindrical vector beam (CVB) generation, using a mode-selective coupler (MSC) as transverse mode converter and splitter. The all-normal-dispersion mode-locked Ytterbium-doped fiber laser operating at DSR regime without spectral filter can deliver CVB pulses with a pulse duration of $\sim 733$ ps and a pulse energy as high as $\sim 0.439 \mathrm{~nJ}$. The radially and azimuthally polarized beams can be switched by adjusting the polarization controller, with a high mode purity measured to be $>94.5 \%$. This compact DSR fiber laser could find potential applications in the field of material processing, nonlinear optics and so on.
\end{abstract}

Keywords: Cylindrical vector beam; Ytterbium-doped fiber laser; dissipative soliton resonance 


\section{INTRODUCTION}

Passively mode-locked fiber lasers based on nonlinear polarization rotation have been widely investigated because of their inherent advantages and attractive properties. Due to the comprehensive interplay among many factors (nonlinear, dispersion, gain and loss), pulses risen in a mode-locked fiber laser usually have rich and complex nonlinear dynamics [1]. Various pulse states [2-4], such as Q-switched pulse, conservative soliton, stretched pulse, dissipative soliton, noise-like pulse, and similariton, have been reported depending on cavity configuration. What is more, the behavior of the pulses can also be single-pulse, multi-pulse, and unstable pulses with periodic or non-periodic fluctuation etc $[5,6]$. Especially, dissipative soliton resonance has been considered as an approach to boost the pulse energy to a higher level [7-9]. Dissipative solitons with steep spectral edges can be attained from all-normal-dispersion fiber lasers through introducing spectrum filter. The spectral filtering effect can balance the strong self-amplitude modulation in the all-normal-dispersion lasers, resulting in mode-locking of highly chirped pulses with unprecedented energies. However, the used spectrum filter inevitably restricts the increase of pulse energy. Fortunately, it has been validated that dissipative soliton resonance can be reachable in all-normal-dispersion fiber laser without spectrum filter $[10]$.

On the other hand, cylindrical vector beams (CVBs) including radial polarization, azimuthal polarization, and hybrid polarization beams, have many potential applications, such as optical trapping, optical communications, surface plasmon excitation, electron acceleration, high resolution metrology, and material processing, due to their unique properties of symmetrical polarization and amplitude [11]. For an instance, radially polarized beams have the ability to focus on smaller spot sizes for 
nanoparticle manipulation, azimuthal polarized beams can generate high magnetic to electric field contrast, i.e., large local field admittance [12]. For the generation of CVBs, optical fiber-based CVB generation method seems more desirable owing to its flexibility, robustness, and compatibility with optical-fiber communication system. The excitation of TM01 and TE01 eigenmodes with cylindrical symmetry in few-mode fiber (FMF), has been investigated mainly through two kinds of techniques: mode selector and convertor. A commonly used mode excitation method is to include offset splicing between a single-mode fiber (SMF) and a FMF to excite multiple higher-order modes, together with an few-mode fiber Bragg grating (FM-FBG) to serve as a mode selector. However, offset splicing inevitably induces an excess loss due to mode field mismatch, and FM-FBG presents a rather narrow bandwidth for mode conversion. The mode-selective coupler (MSC), as a typical mode convertor, has been used to convert the LP01 in the SMF to the LP11 group in the FMF. This method has been applied to quite a few fiber lasers including CW, Q-switched and mode-locked fiber lasers [13-15]. However, most of the works on CVB generation lay emphasis on the CVB pulse output from Erbium-doped fiber lasers, while few report on CVB pulse Ytterbium-doped fiber laser, much less dissipative soliton resonance CVB pulse Ytterbium-doped fiber laser.

In this paper, we propose and demonstrate an all-normal-dispersion mode-locked Ytterbium-doped fiber laser without spectral filter operating at DSR regime delivering CVBs with a MSC for transverse mode conversion and selective output. As a result, both radially and azimuthally polarized beams in DSR mode-locked states have been obtained for the first time from passively mode-locked fiber laser. The purity of both radially and azimuthally polarized beams is measured to be $>94.5 \%$. This DSR 
Ytterbium-doped fiber laser with all-fiber configuration could have potential applications in the field of material processing, nonlinear optics and so on.

\section{EXPERIMENT AND DISCUSSES}

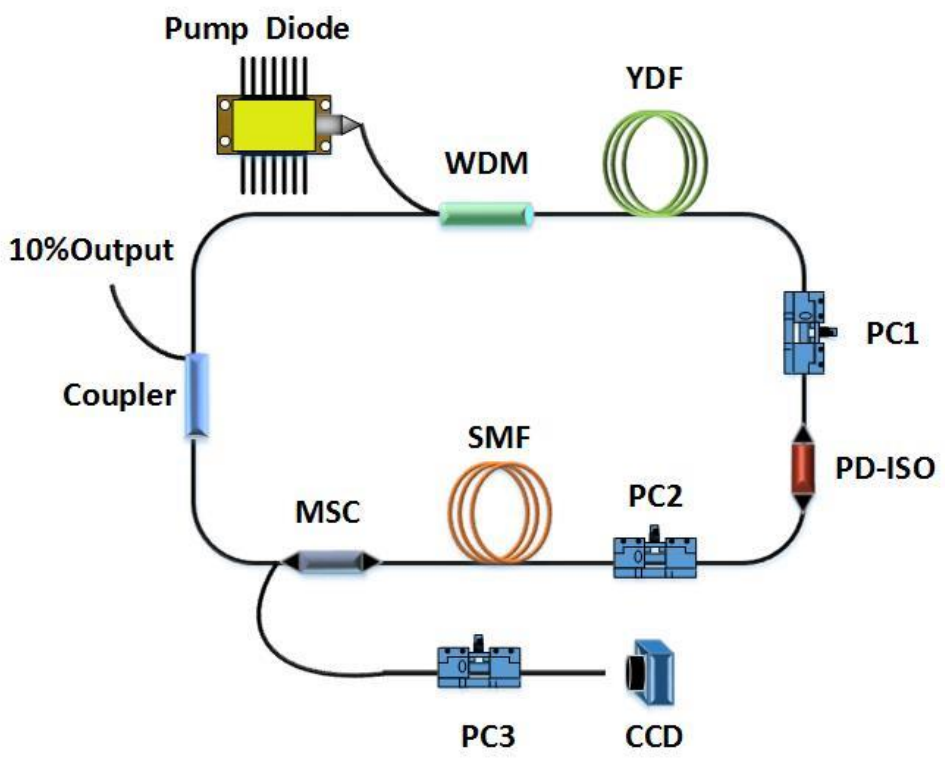

Fig.1 Experimental setup of the proposed mode-locked fiber laser based on nonlinear polarization rotation with CVB generation.

The experimental setup of DSR mode-locked fiber laser based on nonlinear polarization rotation with CVB generation is shown in Fig. 1 . A $1.0 \mathrm{~m}$ Ytterbium-doped fiber (YDF) as high-gain medium has an absorption coefficient of $\sim 250 \mathrm{~dB} / \mathrm{m}$ at $980 \mathrm{~nm}$, which is pumped by a $980 \mathrm{~nm}$ laser diode with the maximum pump power of $700 \mathrm{~mW}$ through a 980/1064 nm wavelength division multiplexing coupler (WDM). A polarization-dependent isolator (PD-ISO) playing double roles of isolating and polarizing is sandwiched between two polarization controllers (PC1 and PC2) for mode-locking. A section of single-mode fiber (SMF) with length of $15 \mathrm{~m}$ is used to manage the nonlinearity and dispersion for DSR operation. The MSC is 
located behind the second PC, i.e. PC2. Another polarization controller (PC3) is added on the MSC pigtail for controlling the CVB generation and extracting radially polarized or azimuthally polarized mode. In addition, a 10/90 coupler is engaged to as a function of the output power with $10 \%$ port. The total ring-cavity length is about $21.2 \mathrm{~m}$. And since both single-mode fiber and Ytterbium-doped fiber have normal dispersion at $1 \mu \mathrm{m}$ wavelength, the fiber laser is working in all-normal dispersion region.

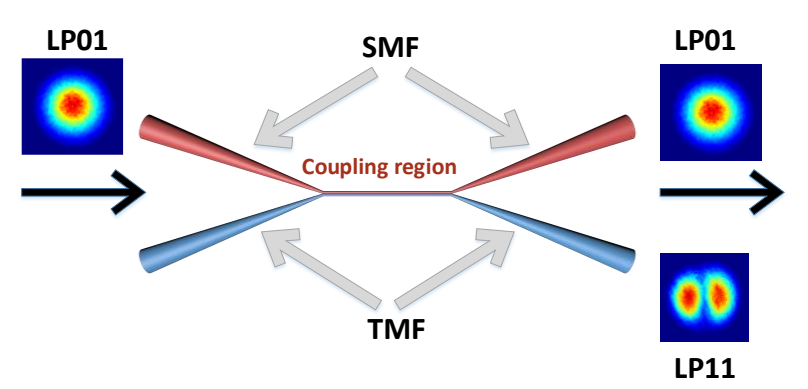

Fig. 2 Schematic of mode-selective coupler

The schematic of the MSC to generate the LP11 mode is shown in Fig. 2. Compared with the offset splicing between SMF and FMF to excite the higher-order modes, MSC as transverse mode converter and splitter has an advantage of reducing the insertion loss and high slope efficiency. The working principle of the MSC is to meet phase-match conditions and maintain the geometry of the two fibers. Thus weak fusion technique is used to ensure accurate phase matching and high mode conversion efficiency. For MSC fabrication, we prepare one conventional single-mode fiber (Hi1060, core/cladding diameter $=6.2 / 125 \mu \mathrm{m}, \mathrm{NA}=0.14)$ and one two-mode fiber $(\mathrm{SMF}-28$, core/cladding diameter $=8.2 / 125 \mathrm{um}, \mathrm{NA}=0.14$ at $1550 \mathrm{~nm})$. The SMF-28 
can be used as the two-mode fiber (TMF) at wavelength of $1064 \mathrm{~nm}$ referring to

$$
\beta=\frac{2 \pi}{\lambda} n_{\text {core }} \sqrt{1-4 \Delta \frac{N+1}{V}}
$$

The SMF was firstly pre-tapered to about $80 \mathrm{um}$, then the well-prepared device was aligned and fused together with TMF using the modified flame method [16]. With the tunable laser source launched into the SMF input, mode intensity distribution at the TMF output port can be detected by a CCD camera (CinCam IR, InGaAs). The loss of our fabricated MSC converting the LP01 mode efficiently to the LP11 mode is as low as $0.5 \mathrm{~dB}$. The purity of the LP11 mode is estimated to be above $96 \%$ at a wavelength of $1064 \mathrm{~nm}$, using the method of the tight bend approach [17].
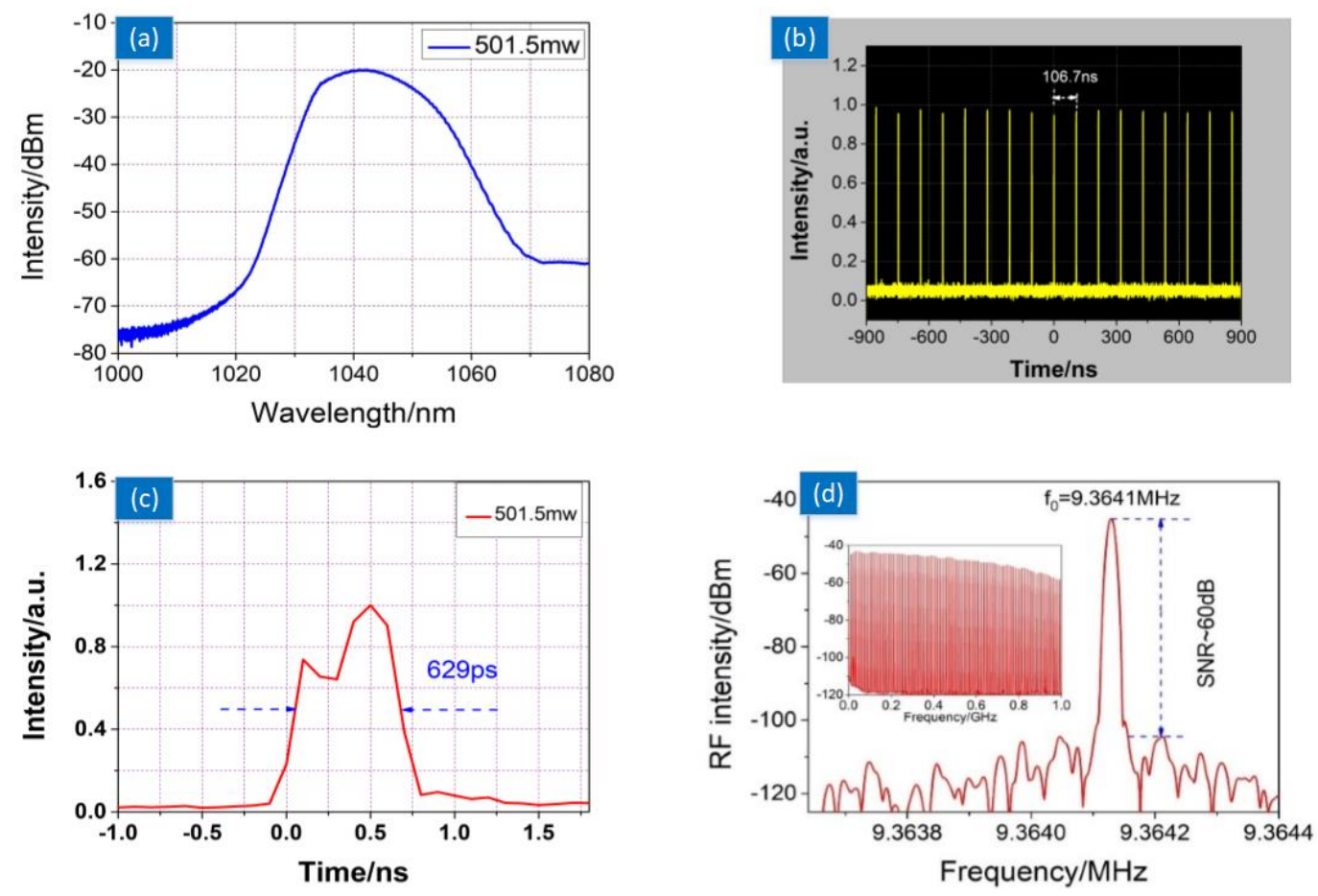

Fig. 3 DSR pulse characteristics at pump power of $501.5 \mathrm{~mW}$. (a) Optical spectra, (b)

oscilloscope trace, (c) single pulse profile, and (d) RF spectrum at the fundamental frequency.

Inset: wideband RF spectrum up to $1 \mathrm{GHz}$. 
In our experiment, the continuous-wave (CW) laser threshold is $98.4 \mathrm{~mW}$. The stable mode-locking can be observed when pump power is above $358.4 \mathrm{~mW}$ by appropriately adjusting the polarization state of the ring cavity through the PC1 and PC2. As shown in Fig. 3(a), the output optical spectrum lasing at $1041.89 \mathrm{~nm}$ has a linewidth of $14.19 \mathrm{~nm}$ at $501.5 \mathrm{~mW}$ pump power, which is similar to a Gaussian profile. The average output power is about $3.35 \mathrm{~mW}$ and the ouput pulse energy is calculated to be $0.358 \mathrm{~nJ}$. The pulse train can be observed in the oscilloscope, as shown in Fig. 3(b). The pulse-to-pulse interval is 106.7 ns, which corresponds to the round trip time of the cavity. Figure 3(c) illustrates the corresponding temporal profile of single pulse, which has an almost rectangular shape with a full width at half maximum (FWHM) of 629 ps. Noted that pulse shape may affected by the sampling rate of the used oscilloscope. Figure 3(d) gives the RF spectrum of the DSR pulses measured under a bandwidth resolution of $10 \mathrm{KHz}$. The signal-to-noise ratio (SNR) of the RF spectrum is more than $60 \mathrm{~dB}$ as the fundamental frequency peak is located at 9.364 MHz, which is in agreement with the theoretical calculation value by the 21.2 m cavity length. Meanwhile, the inset of Fig. 3(d) shows a wideband RF spectrum up to $1 \mathrm{GHz}$ without any modulaion, indicating the stable $\mathrm{CW}$ mode-locking operation in the laser. 

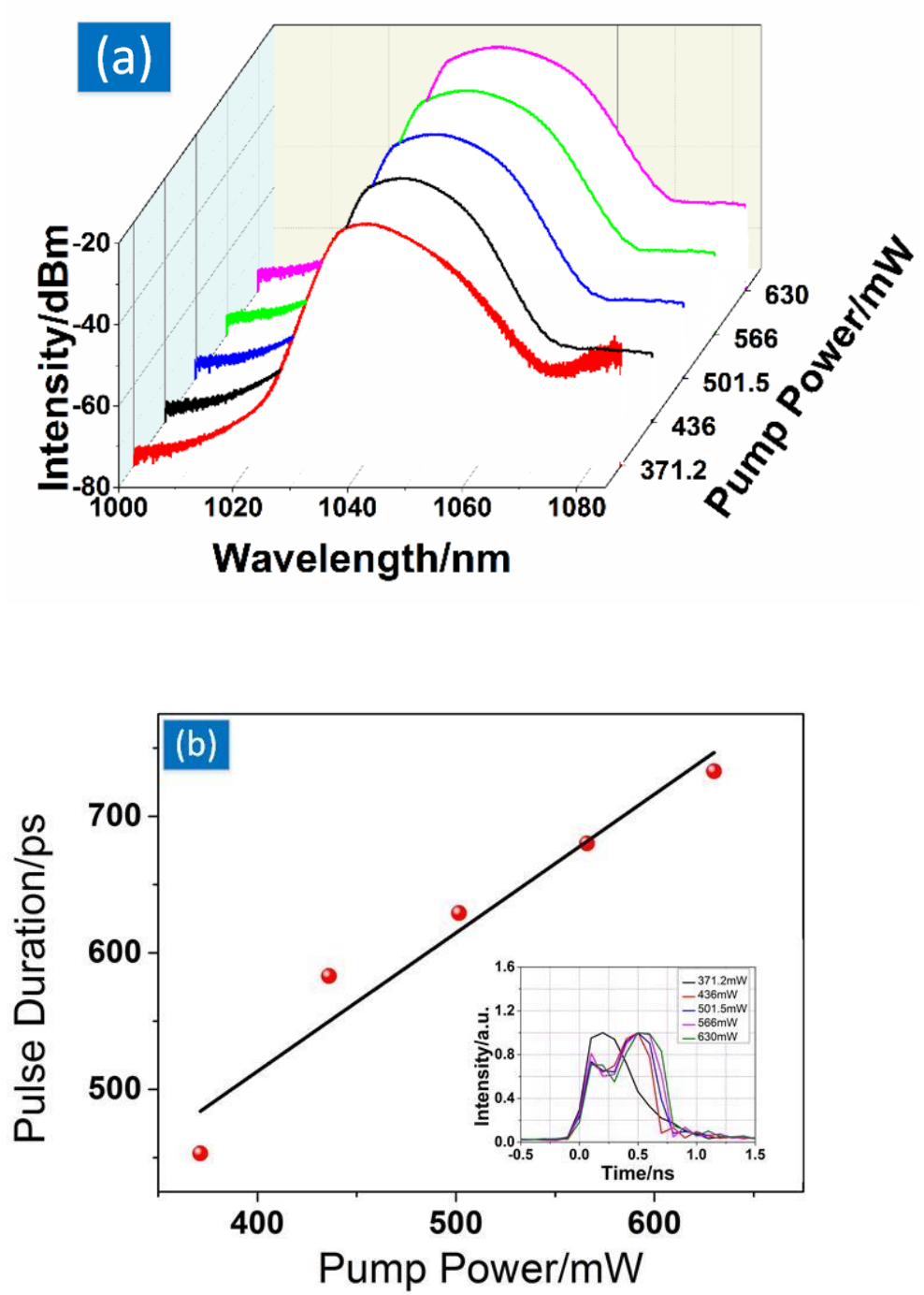

Fig. 4 (a) Optical spectra and (b) single pulse evolution as the pump power increases.

The typical feature of DSR pulses can be validated in our fiber laser as the pump power gradually increased. Fig. 4(a) shows the output optical spectra under different pump powers of $371.2,436,501.5,566$, and $630 \mathrm{~mW}$. As it can be seen, both the spectral profile and the spectral FWHM barely change and remain almost constant, despite of the improved spectral intensity with the increased pump power. The emitted central wavelength is fixed and strain-controlled in the stable ring-cavity 
structure based on PD-ISO with increasing power. Correspondingly, we also measured the temporal profiles of the dissipative soliton corresponding to their optical spectra, as depicted in Fig. 4(b). The single-pulse FWHM are 453, 583, 629, 680, and 733 ps respectively, indicating the rectangular pulse gradually broadens with the increase of incident pump power. The spectral and pulse evolutions are in good agreement with the DSR features reported previously [18-20]. In order to verify the generated pulses are in the DSR regime, the autocorrelation trace has been measured, which does not show any pulse or spike over the maximum measurement range, and suggest the pulse is free of any sub-structure.
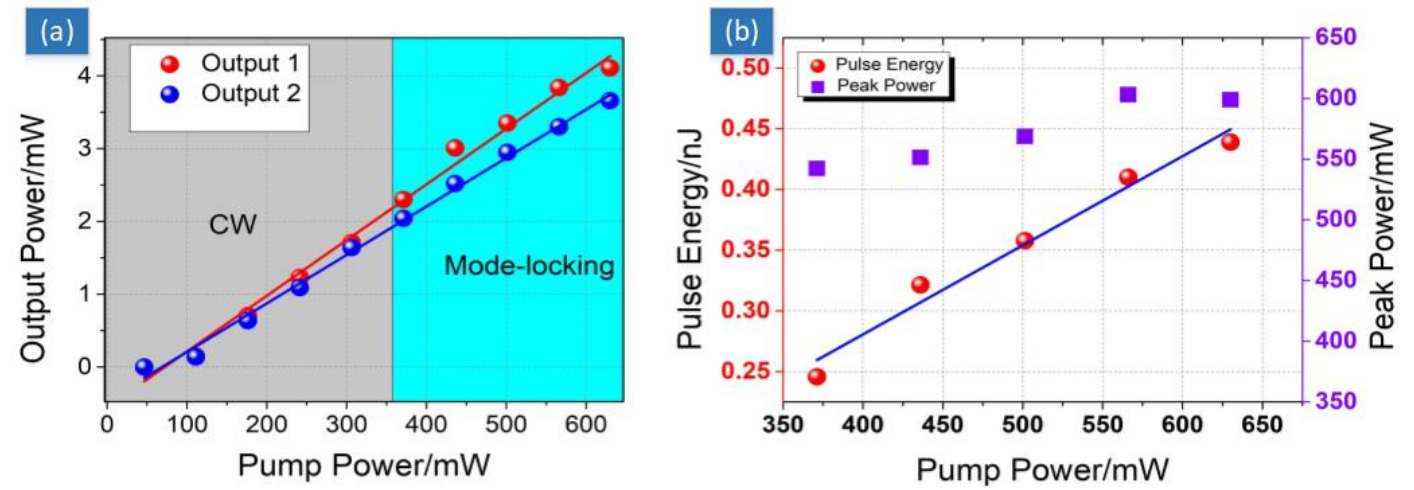

Fig. 5 (a) Output power verse pump power, (b) pulse energy (read line) and peak power (purple line) as functions of pump power

In order to further understand the performance of our fiber laser, we also recorded the average output power (the output 1 representing the 10\% coupler and the output 2 representing the MSC output), pulse energy, and peak power verse the pump power. As seen in Fig. 5(a), when the pump power is increased, the average output power quasi-linearly increased with the incident pump power without the appearance of saturation. The slope efficiency is $0.77 \%$ (output 1) and $0.67 \%$ (output 2) 
respectively. The maximum average output power in stable operation obtained in this experimental setup is $4.11 \mathrm{~mW}$ corresponding to output 1 , and it is limited by the losses of the cavity and pump power. In Fig. 5(b), we correspondingly calculated the pulse energy and peak power using the measured average power at a pulse repetition rate of $9.364 \mathrm{MHz}$. The pulse energy linearly increases from 0.246 to $0.439 \mathrm{~nJ}$ with the pump power increase from 371.2 to $630 \mathrm{~mW}$. Meanwhile, the pulse peak power increases at first but then remains constant due to the periodic saturable absorption of the nonlinear polarization rotation [21], and it can reach a maximum of $\sim 0.6 \mathrm{~W}$.

It is well known that the CVB exhibits a doughnut-shaped intensive pattern. When PC3 is appropriately adjusted, the doughnut-shaped intensity pattern can be monitored in the MSC output port by CCD camera. After passing through a linear polarizer between the collimator and the CCD camera, the radially and azimuthally polarized beams could be discriminated. Fig. 7(a-j) show the mode intensity distributions of the radially and azimuthally polarized beams and what they look like after passing a linear polarizer at different orientations. Furthermore, by adopting a fiber bending method with principle of attenuating radially or azimuthally polarized modes and then evaluating their purity by calculating the percentage bending loss, we estimate that the purities of the radially and azimuthally polarized beam are $95.1 \%$ and $94.5 \%$, respectively. 

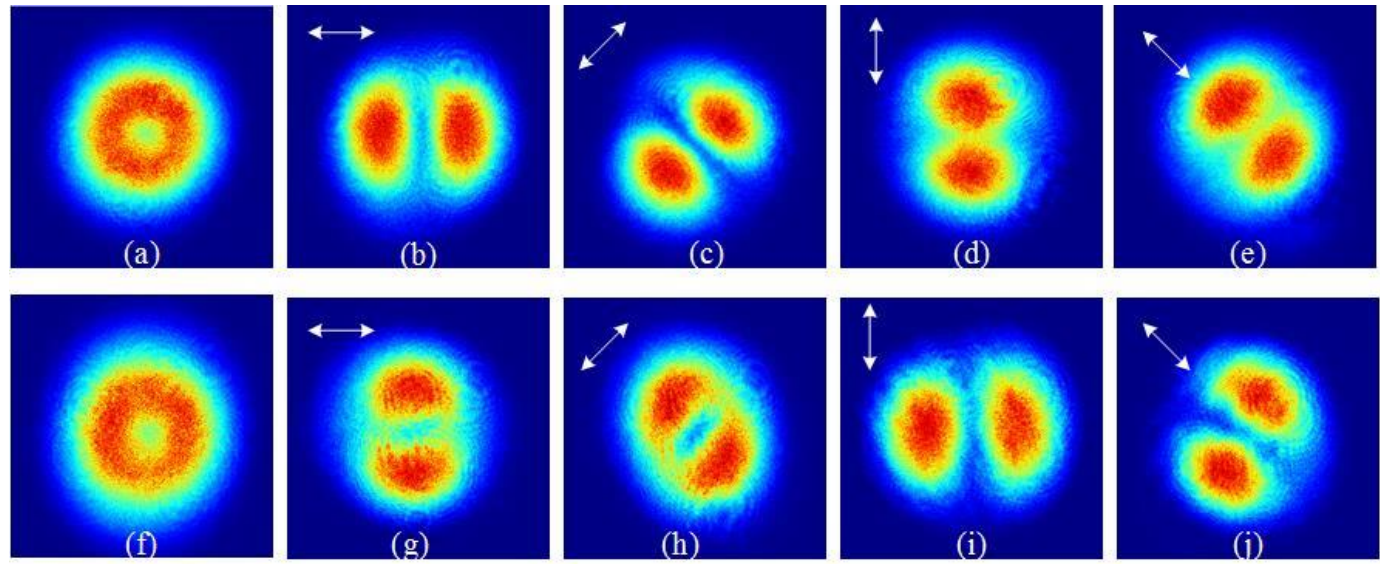

Fig. 6 Intensity distributions of radially (a) and azimuthally (f) polarized beams:

without a polarizer, (b)-(e) and (g)-(j) after passing through a linear polarizer with transmission axis orientation denoted by the arrows.

\section{CONCLUSIONS}

In summary, a DSR pulse ytterbium-doped fiber laser with high-purity cylindrical vector beam generation based on a MSC has been proposed and demonstrated. Through managing the nonlinearity and dispersion of the fiber laser, DSR pulses can be obtained from the all-normal-dispersion ytterbium-doped fiber laser without a spectral filter. CVB conversion and separation are attributed to the MSC consisting of both SMF and few-mode fiber (SMF28, which is single-mode fiber at $1.55 \mu \mathrm{m}$ wavelength band, but few-mode fiber at $1 \mu \mathrm{m}$ wavelength band). CVB pulses with a pulse duration of $\sim 733 \mathrm{ps}$ and a pulse energy as high as $\sim 0.439 \mathrm{~nJ}$ have been achieved, with a high purity measured to be $>94.5 \%$. We believe that this compact DSR fiber laser could find potential applications in material processing, nonlinear optics and other fields.

Acknowledgments: This work is supported by National Science Foundation of 
Jiangsu Province under Grants BK20161521; Nanjing University of Posts and

Telecommunications (NUPTSF) under Grants NY214002, and NY215002;

Distinguished Professor Project of Jiangsu under Grant RK002STP14001; Six Talent

Peaks Project in Jiangsu Province under Grant 2015-XCL-023; Postgraduate Research

\& Practice Innovation Program of Jiangsu Province under Grants KYCX17_0744 and

SJCX17_0234.

\section{References:}

1. Wei H, Li B, Shi W, et al. General description and understanding of the nonlinear dynamics of mode-locked fiber lasers. Sci. Rep. 2017; 7(1):1292.

2. Wang L, Liu X, Gong Y, Mao D and Duan L. Observations of four types of pulses in a fiber laser with large net-normal dispersion. Opt. Express 2011; 19:7616-7624.

3. Peng J, Boscolo S. Filter-Based Dispersion-Managed Versatile Ultrafast Fibre Laser. Sci. Rep. 2016; 6:25995.

4. Liu X. Hysteresis phenomena and multipulse formation of a dissipative system in a passively mode-locked fiber laser. Phys. Rev. A 2010; 81(2):023811

5. Ding E, Shlizerman E, Kutz J N. Generalized master equation for high-energy passive mode-locking: the sinusoidal GinzburgLandau equation. IEEE J. Quantum Electron 2011; 47:705-714.

6. Cabasse A, Ortaç B, MartelG, Hideur A, and Limpert J. Dissipative solitons in a passively mode-locked Er-doped fiber with strong normal dispersion. Opt. Express 2008; 16:19322-19329.

7. Chang W, Ankiewicz A, Soto-Crespo J M, Akhmediev N. Dissipative soliton resonances. Phys. Rev. A 2008; 78:023830.

8. Wu X, Tang D Y, Zhang H, Zhao L M. Dissipative soliton resonance in an all-normal dispersion erbium-doped fiber laser. Opt. Express 2009; 17: 5580-5584.

9. Liu X. Numerical and experimental investigation of dissipative solitons in passively mode-locked fiber lasers with large net-normal-dispersion and high nonlinearity. Opt. Express 2009; 17: 22401-22406.

10. $\mathrm{Xu} \mathrm{Z} \mathrm{W}$, Zhang $\mathrm{Z} \mathrm{X}$. Diverse output states from an all-normal dispersion ytterbium-doped fiber laser: Q-switch, dissipative soliton resonance, and noise-like pulse. Optics \& Laser Technology 2013; 48(6):67-71.

11. Mao D, Feng T, Zhang W. Ultrafast all-fiber based cylindrical-vector beam laser. Appl. Phys. Lett. 2017; 110(2): 021107.

12. Sun B, Wang A, Gu C. Mode-locked all-fiber laser producing radially polarized rectangular pulses. Opt. Lett. 2015; 40(8):1691-4. 
13. Wang T, Wang F, Shi F. Generation of femtosecond optical vortex beams in all-fiber mode-locked fiber laser using mode selective coupler. Journal of Lightwave Technology 2017; 35(11):2161-2166.

14. Wan H, Wang J, Zhang Z, et al. High efficiency mode-locked, cylindrical vector beam fiber laser based on a mode selective coupler. Opt. Express 2017; 25(10):11444-11451.

15. Mao D, He Z, Lu H, et al. An All-fiber radially/azimuthally polarized lasers based on mode coupling of tapered fibers. Opt. lett. 2018; 43(7):590-1593.

16. Wang J, Wan H, Cao H. A $1.0 \mu \mathrm{m}$ Cylindrical Vector Beam Fiber Ring Laser Based on A Mode Selective Coupler. IEEE Photon. Technol. Lett. 2018; 30(9): 765-768.

17. Sun B, Wang A, Xu L, Gu C, Lin Z, Ming H and Zhan Q. Low-threshold single-wavelength all-fiber laser generating cylindrical vector beams using a few-mode fiber Bragg grating. Opt. Lett. 2012; 37(4):464-6.

18. Wang S K, Ning Q Y, Luo A P, et al. Dissipative soliton resonance in a passively mode-locked figure-eight fiber laser. Opt. Express 2013; 21(2):2402-7.

19. Semaan G, Braham F B, Fourmont J, et al. $10 \mu \mathrm{J}$ dissipative soliton resonance square pulse in a dual amplifier figure-of-eight double-clad Er:Yb mode-locked fiber laser. Opt. Lett. 2016; 41(20):4767-4770.

20. Krzempek K, Sotor J, Abramski K. Compact all-fiber figure-9 dissipative soliton resonance mode-locked double-clad Er:Yb laser. Opt. Lett. 2016; 41(21):4995-4998.

21. Li D, Tang D, Zhao L, et al. Mechanism of Dissipative-Soliton-Resonance Generation in Passively Mode-Locked All-Normal-Dispersion Fiber Lasers. Journal of Lightwave Technology 2015; 33(18):3781-3787. 